\title{
B04
}

\section{The Tilt Derivative Applied to AEM Conductivity} Data

\author{
D. Beamish* (British Geological Survey)
}

DOI: $10.3997 / 2214-4609.20147029$

\section{SUMMARY}

Various procedures are used in the processing of potential field data to provide enhanced detection and definition of structural information. The majority of such procedures use the spatial derivatives of the data either individually or in combination. The ability of the tilt angle (or derivative) to provide enhanced mapping of electromagnetic (EM)/conductivity structure is considered here. Although this study considers airborne EM survey data, the concept can equally be applied to ground-based conductivity/resistivity data sets. Following a description of the principles of the tilt derivative (TDR) method, forward modelling studies of concealed EM/conductivity structure are presented. Case studies of the practical application of the procedures to survey data are then performed. The tilt function embodies Automatic Gain Control that normalises the detection and definition of both weak and strong conductivity gradients across an appropriate subsurface depth range. Noise amplification is an inevitable outcome of the procedure. Filtering methods to attenuate undesired artefacts are available and are demonstrated. 


\section{Introduction}

Many of the processing and interpretation methods developed for potential field data sets are based on their spatial derivatives. There now exists a wide range of processing procedures and filters that perform as enhanced mapping functions. The processing procedures now extend the use of vertical and horizontal derivatives and their combinations (e.g. Cooper and Cowan, 2006) and consider higher order derivatives in the search for higher structural resolution. For fields obeying Laplace's equation, filtering procedures, such as Euler deconvolution, are available to provide information on the position and depths of sources.

The ability of the tilt angle (Millar and Singh, 1994) to provide enhanced mapping of resistivity/conductivity structure is considered here. Although the study considers airborne EM survey data, the concept can equally be applied to ground-based conductivity/resistivity data sets. In the case of potential field data, the derivatives are calculated using the observed fields, although in the case of magnetic fields, the data may be first transformed by reduction to the pole/equator. Frequency-domain airborne EM data comprise field coupling ratios that possess a strong dependence on altitude. To avoid this spatial complexity, it is simpler to work with the coupling ratios converted to an equivalent half-space conductivity which are then independent of altitude.

The field interactions involved in EM induction within a 3D conductivity contrast are profoundly more complex than those occurring in the corresponding magnetic susceptibility/density case. Although it is known that in the limit of low frequency and very high contrast targets, the EM wave equation may approximate Laplace's equation, the general inductive case is considered here. The present study, based on conductivity map information, has a general validity, although it should be acknowledged that different geophysical systems (including frequency and time domain systems together with their specific bandwidths) will provide different EM interactions with specific 3D targets.

\section{The tilt derivative method}

The tilt angle/derivative (TDR) is defined by Millar and Singh (1994) as the ratio of a vertical to a combined horizontal derivative:

$$
T D R=\tan ^{-1}\left[\frac{\partial f / \partial z}{\sqrt{(\partial f / \partial x)^{2}+(\partial f / \partial y)^{2}}}\right]
$$

Where $\mathrm{f}$ is the magnetic or gravity field. More recently, Verduzco et al. (2004) suggested using the total horizontal derivative (THDR) of the tilt angle, as an improved edge detector:

$$
T H D R=\sqrt{(\partial T / \partial x)^{2}+(\partial T / \partial y)^{2}}
$$

The amplitude range of the TDR is restricted to the range $\pi / 2$ to $-\pi / 2$ (or $90^{\circ}$ to $-90^{\circ}$ ) by virtue of the arctan function. Both TDR and THDR act as an Automatic Gain Control (AGC) filter when applied to the field observations. This is an important feature when considering its application to larger scale data sets with a wide dynamic range. As noted by Cooper and Cowan (2006), as the order of the derivative function increases, in the search for higher resolution, the degree of noise amplification also increases. The signal/noise content of the

Near Surface $2009-15^{\text {th }}$ European Meeting of Environmental and Engineering Geophysics Dublin, Ireland, 7 - 9 September 2009 
data considered is thus an important aspect in relation to the practical application of the procedures. Summaries of the behaviour of tilt derivatives in the case of potential field data are provided by Fairhead et al. (2004) and Cooper and Cowan (2006).

\section{A synthetic modelling study}

In order to evaluate the performance of tilt derivatives in relation to EM conductivity data we consider the AEM-05 airborne EM system described by Leväniemi et al. (2009). The 4 frequency system comprises a vertical coplanar coil set, mounted across the wing-tips of a DHC Twin-Otter. The 4 frequencies provide different depth ranges of investigation. The lowest frequency is $912 \mathrm{~Hz}$ and this provides the greatest depth of penetration. 3D EM modelling of thin-plate conductivity structures offers the simplest and, typically, the most stable set of solutions for the forward modelling case. Here the thin-plate modelling algorithm LeroiAir developed by the CSIRO Electromagnetic Modelling Group is used for the computations. A single vertical thin-plate with a width of $100 \mathrm{~m}$ and a length of $1.5 \mathrm{~km}$ was embedded in a uniform host of $5 \mathrm{mS} / \mathrm{m}$. The conductance of the plate was $10 \mathrm{~S} . \mathrm{m}$ and the survey height was $50 \mathrm{~m}$. By using a large plate length, it is possible to generate profiles and grids over the central area of the model (e.g. $200 \times 200 \mathrm{~m}$ ) free from the 3D edge effects of a more compact body. Computations were performed with the depth of the plate (the upper surface) increasing from the near-surface to a depth of $150 \mathrm{~m}$. The coupling ratios calculated from the forward modelling at $912 \mathrm{~Hz}$ were converted to half-space apparent conductivities in the standard manner. Figure 1a shows the results obtained across a profile through the centre of the model with the vertical plate located below the origin. The detection amplitudes of the plate decrease with increasing depth and range from $45 \mathrm{mS} / \mathrm{m}$ for the shallowest depth to 1.5 $\mathrm{mS} / \mathrm{m}$ with the plate at a depth of $150 \mathrm{~m}$.

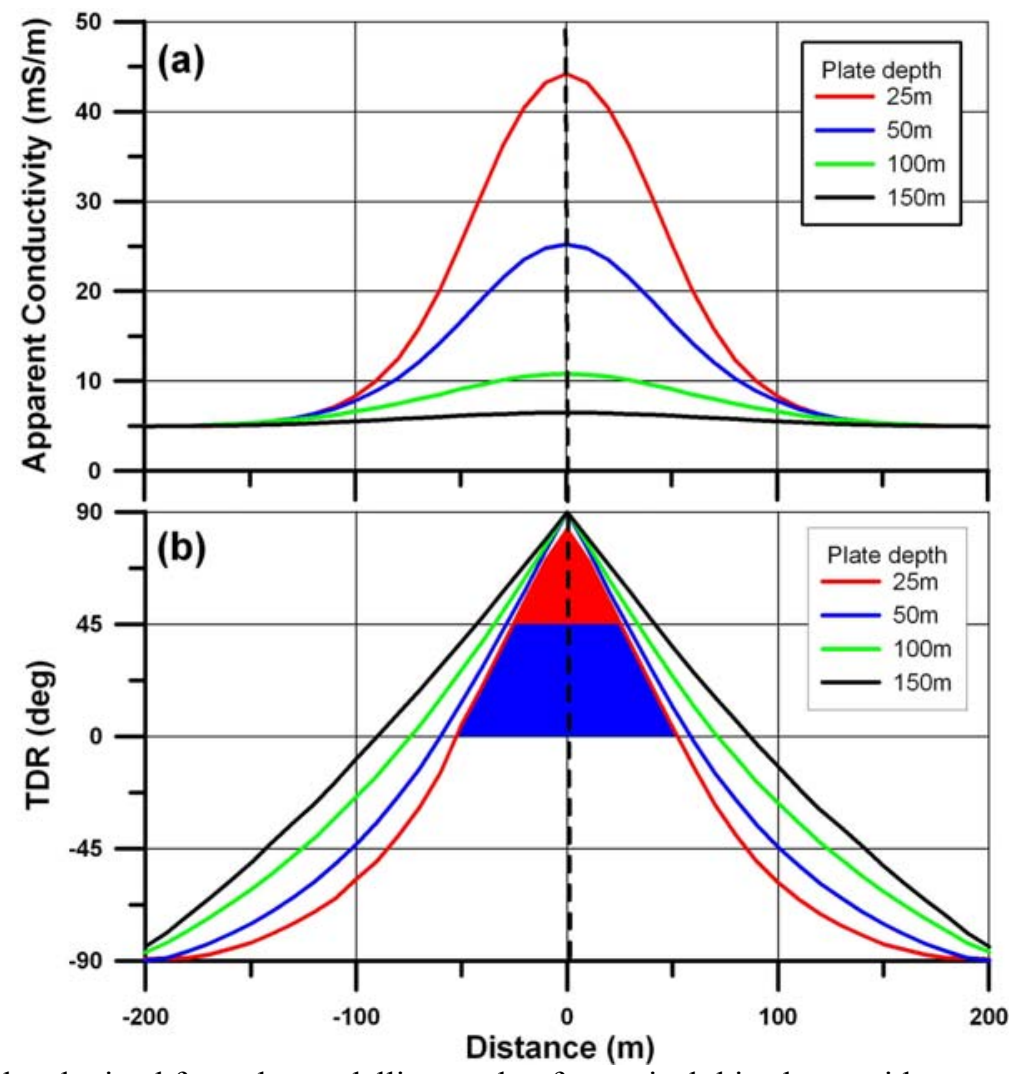

Figure 1. Results obtained from the modelling study of a vertical thin sheet, with an upper surface at the depths shown. (a) Apparent conductivity. (b) Tilt Derivative (TDR). 
The TDR is shown in Figure 1b. The responses display the expected uniform gain at all plate depths, with a value of $+90^{\circ}$ achieved over the centre. If a reference value of $+45^{\circ}$ is chosen as a reference TDR level (red infill) then the location of plate is well defined for plates at shallow depths (e.g. $25 \mathrm{~m}$ ). As the plate increases in depth to $150 \mathrm{~m}$, the assessment of the target location increases to a width of some $+/-45 \mathrm{~m}$. The total horizontal derivative (THDR) of the tilt angle displays a complex ' $\mathrm{M}$ ' shaped behavior across the target, and the simpler form of the TDR is preferred with a mapping of conductive zones using the TDR values from 0 to $45^{\circ}$ (blue) and 45 to $90^{\circ}$ (red).

\section{Survey data example}

A selected example of the application of the THDR method to conductivity mapping uses half-space conductivity data from the Tellus airborne geophysical survey of Northern Ireland (Beamish and Leväniemi, 2006). The example is a $6 \times 6 \mathrm{~km}$ area taken across a zone of Variscan deformation containing, and to the north of, the Omagh Thrust Fault zone. The major part of the area contains the Lack inlier (Dalradian metasediments) which is bounded to the north by the Cool fault. The survey detected strong conductive features associated with the fault zones and a further less conductive feature (labeled X in Figure 2b) within the inlier.
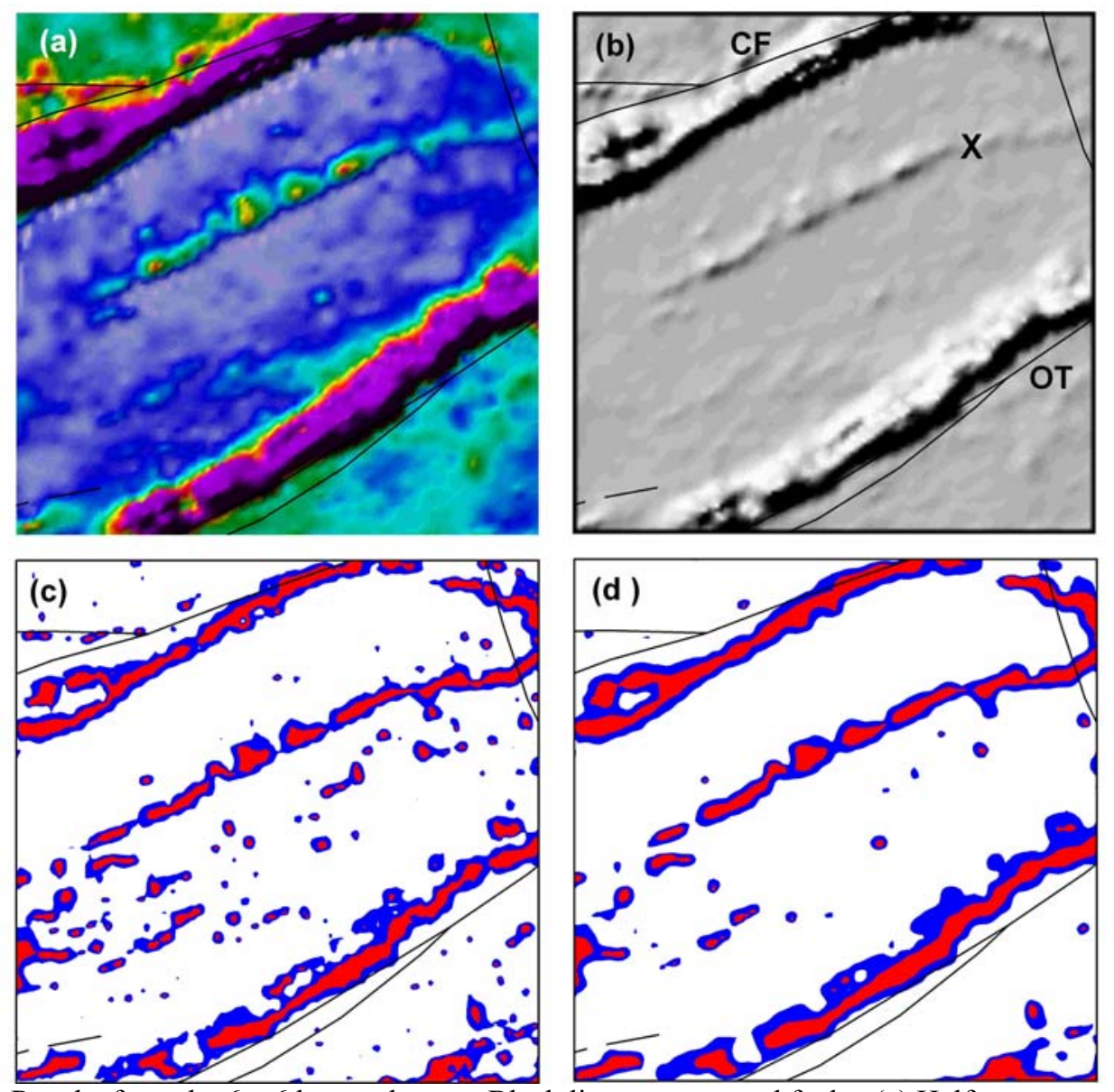

Figure 2. Results from the $6 \times 6 \mathrm{~km}$ study area. Black lines are mapped faults. (a) Half-space conductivity at $3 \mathrm{kHz}$. (b) Shaded relief image of the conductivity data. (c) TDR of data in (a). (d) TDR of data upward continued by $50 \mathrm{~m} . \mathrm{CF}=\mathrm{Cool}$ fault. OT=Omagh Thrust. $\mathrm{X}=$ Unmapped lineament.

Figure 2a shows the apparent conductivity data obtained at a frequency of $3 \mathrm{kHz}$ across the 6 x $6 \mathrm{~km}$ area. The colour scale is linear and ranges from 0 (grey) to 25 (purple) $\mathrm{mS} / \mathrm{m}$. Sun

Near Surface $2009-15^{\text {th }}$ European Meeting of Environmental and Engineering Geophysics Dublin, Ireland, 7 - 9 September 2009 
shading from the NW has been applied to emphasise the conductivity gradients. The shaded relief image of the data is shown in Figure 2b. Figure $2 \mathrm{c}$ shows the TDR calculated from the data with only positive values displayed. Blue corresponds to the TDR range $0^{\circ}$ to $45^{\circ}$ and red corresponds to the range $45^{\circ}$ to $90^{\circ}$.

The results also demonstrate the noise amplification inherent in the procedure. A large number of high-wavenumber features are clearly present in the TDR map. When applied to other data sets from the UK, the sensitivity of the procedure to small scale conductivity gradients such as those associated with roads is well demonstrated. Although some of these features may be of interest in relation to local-scale environmental investigations, it is worth demonstrating the application of another form of filtering procedure, common in potential field processing, to the removal of the high wave-number content. Although a number of potential low-pass filters could be applied to the conductivity data, the method of upwardcontinuation is demonstrated in Figure 2d. The optimum height for upward-continuation is a matter of experimentation, the conductivity data of Figure 2a were first upward continued to a height of $50 \mathrm{~m}$. Figure $2 \mathrm{~d}$ then shows the resulting TDR map calculated from the data and is shown using the same colour ranges as previously. The results indicate the ability of the procedure to detect and outline significant conductive features throughout the subsurface.

\section{Conclusions}

This study has considered the application of the total horizontal derivative of the tilt angle to enhance half-space conductivity mapping. In the airborne case, the lowest frequency (or latest time) offers the greatest potential for summarising the outlines of conductive domains across the largest range of depths. The inherent AGC of the technique normalises the detection and definition of both strong and weak subsurface conductive features. An equivalent procedure to map resistive features could equally be applied to any geophysical data sets mapped in terms of their resistivity.

\section{References}

Beamish, D. and Leväniemi, H. [2006] The Tellus airborne geophysical survey of Northern Ireland. EAGE Near Surface 2006 Conference, Helsinki, Extended Abstracts, B022.

Cooper, G. R. J. and Cowan D.R. [2006] Enhancing potential field data using

filters based on the local phase. Computers and Geosciences, 32, 1585-1591.

Fairhead, J.D., Green, C.M., Verduzco, B. and Mackenzie, C. [2004] A new set of magnetic field derivatives for mapping mineral prospects. ASEG $17^{\text {th }}$ Geophysical Conference and Exhibition, Sydney, Extended Abstracts.

Leväniemi, H., Beamish, D., Hautaniemi, H., Kurimo, M., Suppala, I., Vironmäki, J., Cuss, R.J., Lahti, M. and Tartaras, E. [2009] The JAC airborne EM system AEM-05. Journal of Applied Geophysics, 67, 219-233.

Miller, H. G., and Singh V. [1994] Potential field tilt - A new concept for location of potential field sources. Journal of Applied Geophysics, 32, 213-217.

Verduzco, B., Fairhead, J.D. and Green, C.M. [2004] New insights into magnetic derivatives for structural mapping. The Leading Edge 23 (2), 116-119. 